\title{
Health Worker Compliance with Infection Prevention and Control Policy in Ghana: A Case Study of La General Hospital
}

\author{
Vincentia D. Kondor ${ }^{1 *}$, Augustine Adomah-Afari ${ }^{2}$ \\ ${ }^{1}$ Health Policy, Planning and Management, School of Public Health, College of Health \\ Sciences, University of Ghana, Legon, Ghana \\ ${ }^{2}$ Lecturer, Health Policy, Planning and Management, School of Public Health, College of \\ Health Sciences, University of Ghana, Legon, Ghana
}

\begin{abstract}
Infection prevention and control is an important topic in the delivery of quality health care because it aims at protecting the patient, the health worker, and the general public. The main objective of the study was to determine health workers' compliance with Infection Prevention and Control policy guidelines during health care delivery. A cross-sectional design using a quantitative method was used to carry out this study in which 143 health workers at the La General Hospital were selected using a stratified sampling method. The data collected using a structured questionnaire was statistically analyzed using chi-square test and logistic regression to establish an association between the dependent and independent variables using a significance level of $p<0.05$ at $95 \%$ Confidence Interval. The results indicated that knowledge of Infection Prevention and Control among health workers was high as $97 \%$ had adequate knowledge, with $64 \%$ of this proportion having excellent knowledge. Additionally, the availability of infection prevention resources to health workers always was 31.4\%; non-compliance due to time constraints at work was $66.4 \%$, and compliance with Infection Prevention and Control guidelines was 30.7\%. Time constraint at work was significantly associated with health workers' compliance with Infection Prevention and Control $(p=0.001)$. The paper suggests that regular in-service training on Infection Prevention and Control must be continued to improve the standards of health delivery practice. Management is encouraged to improve the availability of Infection Prevention resources to health workers and to duly regulate staff workload in order to reduce time constraints and prevent burnout.
\end{abstract}

Keywords: Compliance, Health worker, Healthcare-associated infections, Infection prevention, Knowledge, Standard precautions.

\section{Introduction}

Although Infection Prevention and Control (IPC) precautions have shown to be effective in the prevention, spread, and control of infections, compliance among health workers is low [1]. This could be explained by the fact that although many health workers are well informed about IPC measures, most of them do not appear to follow the IPC guidelines all the time [2]. Crucially, non-compliance with the IPC protocols among health workers leads to the spread of infections within health care settings [3]. Health Care-Associated Infections (HCAI) occur as a result of failure to observe IPC policy guidelines during healthcare interventions [4], and the World Health Organization estimates that millions of patients get infected worldwide [3]. These infections can manifest within the healthcare facility or after the patient is discharged from the hospital. The impact of HCAI includes causing additional trauma and relative disability to patients, increased microbial resistance, 
prolonged hospital stays, increased cost of treatment to patients and relatives and the worst being death [5]. The prevalence of HCAI and its burden affects patients, relatives, health institutions, and other stakeholders such as insurers, hence the need for infection prevention and control measures and policies to regulate these measures.

The IPC policy guidelines/measures include hand washing, wearing of gloves and facemasks, sharps safety, waste management, respiratory hygiene, among others [6]. Washing of hands and changing of gloves between patient contacts serves as a barrier to the transfer of microorganisms from one patient to another as well as to other health workers and health delivery equipment. Despite the introduction of the IPC policy, the prevalence of healthcare-associated infections remains high [7]. Thus, studies examined why infection transmission was still high in health care settings [8]. In their study, [8] found that most health workers did not comply with the IPC policy due to reasons such as non-availability of IPC resources, inadequate knowledge of IPC, allergic reaction to some IPC resources, inadequate staffing, among others.

In the context of Ghana, the infection prevention and control (IPC) policy was formulated to guide the practice of health workers in order to prevent and control the spread of infections within the health care settings; and to solve the problem of healthcareassociated infections, which result from the poor observance of IPC measures during health care delivery [9]. A study conducted at the Ridge Hospital in Ghana found that knowledge of and compliance with IPC among health workers was moderate and called for more efforts to increase health worker knowledge and compliance with IPC [10]. According to [11], the estimated prevalence of healthcareassociated infections in Ghana was high, but then the problem hardly got any attention since it was often not noticed as a problem arising from the health care setting. Some anecdotal reports, such as a news report on Joy FM (a radio station in Ghana) of a father who lost two newborn babies due to infections in a hospital, also suggested a high prevalence of healthcareassociated infections in Ghana [12].

A World Health Organization document observes that very few countries have adequate surveillance systems for healthcare-associated infections, and even most of these countries struggle with its complexity and also lack standardized diagnostic criteria [3].

This study aimed to examine factors associated with infection prevention and control (IPC) policy/guidelines in Ghana by determining health workers' compliance with infection prevention control policy in Ghana. This was achieved by examining the level of knowledge of health personnel of the IPC policy; assessing the availability of IPC resources; and ascertaining if time constraints at work could affect health workers' practice of standard precautions - at the La General Hospital, Greater Accra Region. This paper suggests that policy-makers and stakeholders in the health sector should seek to address these issues in order to help provide information on the extent to which health workers adhere to IPC standard precautions, factors that could be contributing to non-compliance as well as healthcare-associated infections.

\section{Materials and Methods}

The study was carried out at the La General Hospital, a 161-patient bed-capacity hospital located in the La Dadekotopon Municipality of the Greater Accra Region of Ghana. The hospital has 421 health workers comprising anaesthetists, doctors, nurses, pharmacists, orderlies, laboratory technicians, among others. There are 39 units/departments in the hospital, including specialist clinics such as diabetic clinics, physiotherapy, among others.

Data for the study were collected between May and June 2018. A cross-sectional design was used where 143 health workers comprising; doctors, nurses, anaesthetists, orderlies, among 
others, were recruited using both stratified and random sampling methods. A structured questionnaire, which was self-administered by the participants, was used to collect data on socio-demographic characteristics, knowledge of infection prevention and control (IPC), availability of infection prevention and control resources, and compliance with infection prevention and control policy guidelines among health workers.

A composite score was used to measure the relationship between health worker compliance with IPC policy (dependent variable) and health worker knowledge of IPC standard precautions, availability of IPC resources/equipment, and time constraint at work (independent variables). Stata version 15, a data analysis software, was used to analyze the data for frequencies of health workers who had adequate information about IPC policy and guidelines, always had access to the needed IPC resources, had time constraints at work, and always applied standard precaution measures in their health care delivery practices. A chi-square test of association was used to assess the relationship between the dependent and independent variables. The strength of association was confirmed by using multivariate logistic regression analysis. The level of significance was accepted at $\mathrm{p}<0.05$.

\section{Results}

\section{Socio-demographic Characteristics of Respondents}

Out of the estimated 143 health workers, 140 returned the questionnaires giving a response rate of $97.9 \%(140 / 143)$, of which $64 \%(n=89)$ were females, and $36 \%(n=51)$ were males. The mean age of the health workers was 32.1 years $(\mathrm{SD}=8.31)$. Among the respondents, $70 \%$ had a tertiary level of education. $58.8 \%$ were nurses, $17.6 \%$ were paramedics, and $10.7 \%$ were prescribers (doctors and physician assistants). About $43 \%$ had 1-5 years of working experience, with nearly two-fifths (38.4\%) having 6-10 years of working experience. Additionally, $15.0 \%$ had 11-20 years of working experience, while those with $20-40$ years of working experience constituted $3.8 \%$. The results are shown in Table 1 .

Table 1. Socio-demographic Characteristics of Respondents

\begin{tabular}{|l|l|l|}
\hline Characteristics & Frequency & Percentage (\%) \\
\hline Sex $(\mathbf{n}=\mathbf{1 3 9})$ & 50 & 35.97 \\
\hline Male & 89 & 64.03 \\
\hline Female & $(32.13,8.31)$ & - \\
\hline Age $($ mean, $s d)$ & 28 & 20.29 \\
\hline Highest level of Education $(\mathbf{n}=\mathbf{1 3 8})$ \\
\hline None & 28 & 00.00 \\
\hline Primary/Basic & 0 & 6.52 \\
\hline Secondary & 9 & 3.62 \\
\hline Post-Secondary & 5 & 69.57 \\
\hline Tertiary & 96 & 10.69 \\
\hline Professional category $(\mathbf{n}=131)$ & 58.78 \\
\hline Prescribers & 14 & 12.98 \\
\hline Nursing & 77 & 17.56 \\
\hline Laboratory Technician & 17 & 42.86 \\
\hline Paramedical & \\
\hline Years of experience $(\mathbf{n}=\mathbf{1 3 3})$ & 23 & \\
\hline 1-5yrs & 57 & \\
\hline
\end{tabular}




\begin{tabular}{|l|l|l|}
\hline $6-10 \mathrm{yrs}$ & 51 & 38.35 \\
\hline $11-20 \mathrm{yrs}$ & 20 & 15.04 \\
\hline $20-40 \mathrm{yrs}$ & 5 & 3.76 \\
\hline
\end{tabular}

\section{Health Worker Knowledge of Infection} Prevention and Control (IPC) Guidelines

The results showed that 136 (97\%) had adequate knowledge of IPC measures. Of this number, $64 \%$ had excellent knowledge of infection prevention and control guidelines, with 33\% having adequate knowledge and 3\% having poor knowledge of IPC guidelines. Respondents who had had IPC training were $128(94.1 \%)$, and $98.5 \%$ knew that infection could be transmitted through contact with blood and body fluids. In addition, respondents who were aware that hand washing was the single most effective infection prevention and control standard precaution was $125(89.3 \%)$. About 93.6\% (131) of the respondents indicated awareness of the fact that a health worker could transmit infections to patients while attending to them. The results are detailed in Figure 1.

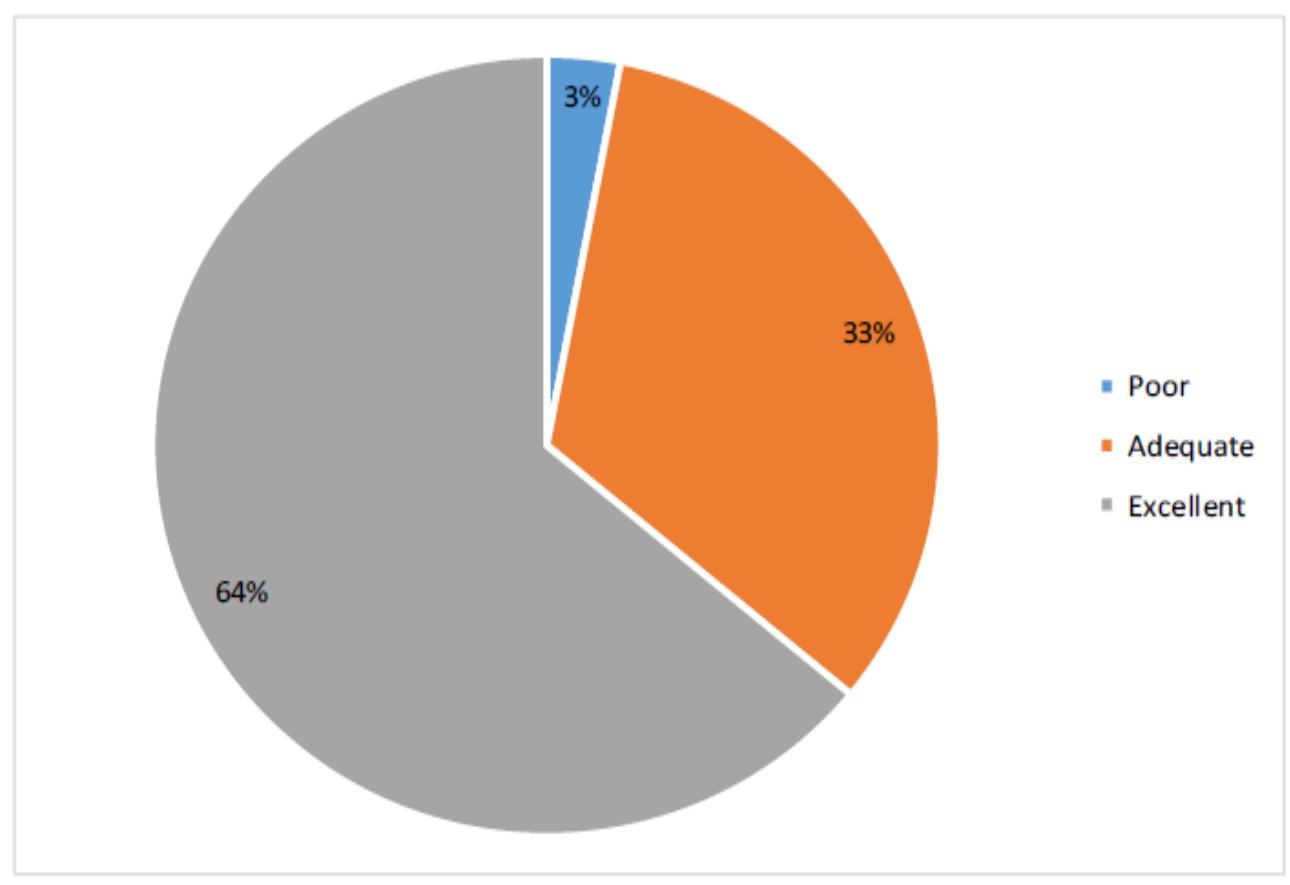

Figure 1. Health Worker Knowledge of Infection Prevention and Control (IPC) Guidelines

\section{Availability of IPC Resources/ Equipment to Health Workers}

The level of awareness of the availability of infection prevention and control (IPC) resources was assessed among the health workers. The results showed that while 44 $(31.4 \%)$ indicated that they were aware that IPC resources were always available, 96 (68.6\%) indicated that IPC resources were not always available. Respondents who indicated that water was always available for hand-washing constituted $6.5 \%$ whilst $93.5 \%$ indicated that water was only available sometimes. Soap for hand-washing was always available (9.4\%), sometimes available (89.2\%), and never available (1.4\%). Availability of hand sanitizer was always available (27.3\%) and sometimes available (69.8\%). Figure 2 below shows the results of the availability of IPC resources. 


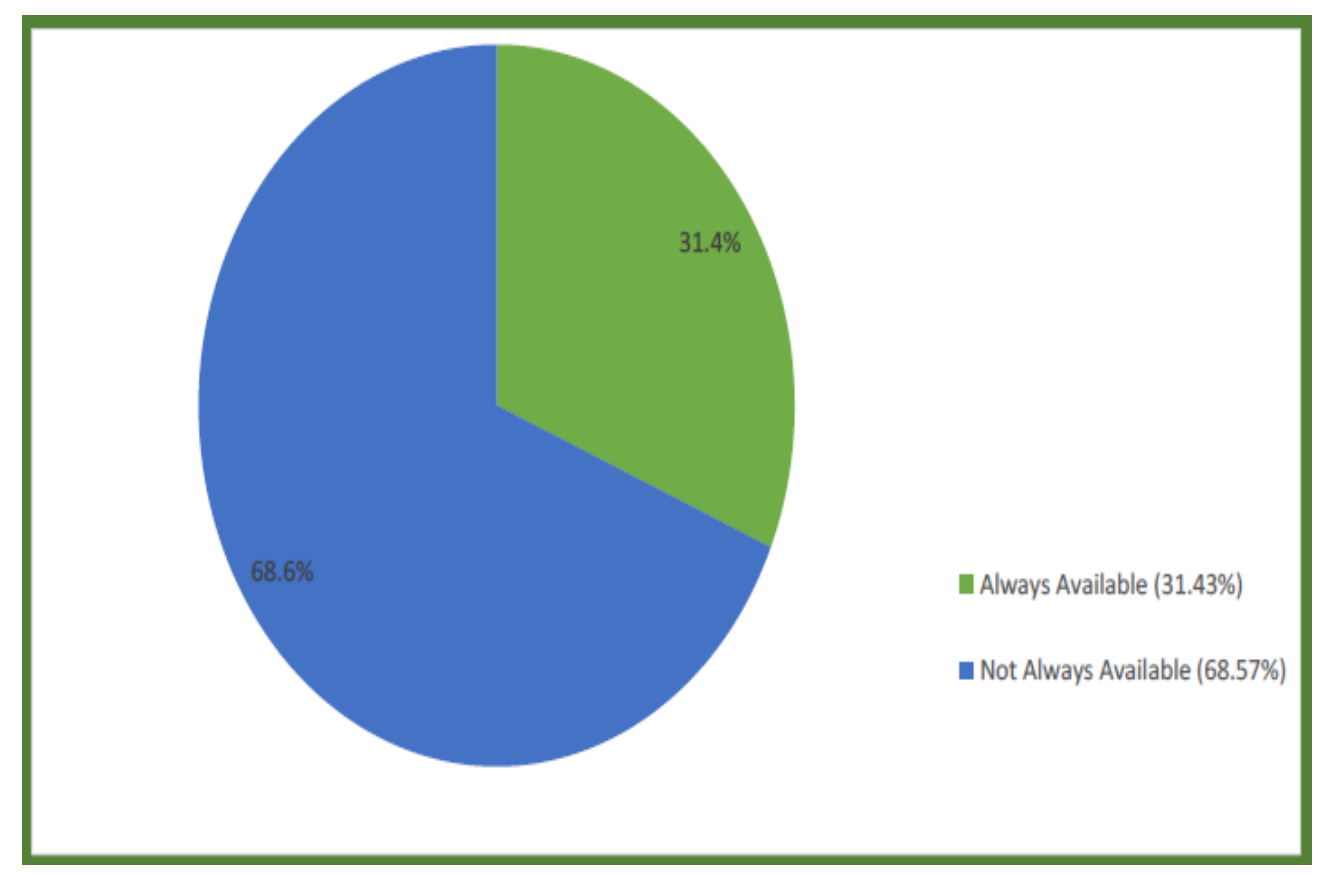

Figure 2. Availability of IPC Resources/ Equipment to Health Workers

\section{Health Workers' Compliance with IPC Policy Guidelines}

Respondents' level of compliance with infection prevention and control policy guidelines was $30.7 \%$ (43/140), whereas noncompliance was $69.3 \%$ (97/140). Only 70
(57\%) stated that they do wash their hands before touching patients. Moreover, $73.1 \%$ of respondents indicated that they always wear hand gloves before doing a procedure that had a risk of exposure to body fluids. The results are shown in Table 2 below.

Table 2. Health Workers' Compliant with IPC Policy Guidelines

\begin{tabular}{|l|l|l|}
\hline Resource availability $(\mathbf{n = 1 4 0})$ & Number & Percentage \\
\hline Noncompliant & 97 & 69.3 \\
\hline Compliant & 43 & 30.7 \\
\hline Total & $\mathbf{1 4 0}$ & $\mathbf{1 0 0}$ \\
\hline
\end{tabular}

\section{Time Constraint at Work}

The results showed that 89 (66.4\%) out of 134 respondents indicated non-compliance with IPC guidelines due to time constraints at work. Of this number, 28 (20.8\%) always had time constraints hindering their adherence to hand hygiene practices, while $61(45.5 \%)$ were hindered sometimes. The results are shown in Table 3.

Table 3. Time Constraint at Work

\begin{tabular}{|l|l|l|}
\hline Time Constraint at Work $(\mathbf{n}=\mathbf{1 3 4})$ & Number & Percentage $(\boldsymbol{\%})$ \\
\hline Always & 28 & 20.9 \\
\hline Sometimes & 61 & 45.5 \\
\hline Never & 45 & 33.6 \\
\hline Total & $\mathbf{1 3 4}$ & $\mathbf{1 0 0}$ \\
\hline
\end{tabular}


Chi-Square Test: Relationship between Predictor Variables and Compliance with IPC Policy Guidelines

The Chi-square test of association was used to measure the relationship between the predictor variables (sex, educational level, professional category, years of experience, time constraint at work, availability of resources, and level of knowledge) and the outcome variable (health workers' compliance with IPC policy guidelines). The test was carried out at a 95\% confidence interval. The results revealed that time constraints at work were significantly associated with compliance with IPC policy guidelines $(p=0.001)$. All other variables were not significantly associated. The results are shown in Table 4 below.

Table 4. Chi-square Test: Relationship between Predictor Variables and Compliance with IPC Policy Guidelines

\begin{tabular}{|c|c|c|c|c|c|c|}
\hline \multicolumn{7}{|c|}{ Compliance with IPC policy guidelines } \\
\hline \multirow{2}{*}{ Variables } & \multicolumn{2}{|c|}{ Noncompliant } & \multicolumn{2}{|c|}{ Compliant } & \multirow[t]{2}{*}{$\chi^{2}$} & \multirow[t]{2}{*}{ p - value } \\
\hline & Number & $\%$ & Number & $\%$ & & \\
\hline Sex & \multicolumn{2}{|l|}{$\mathrm{N}=97$} & \multicolumn{2}{|l|}{$\mathrm{N}=42$} & & \\
\hline Male & 33 & 66.00 & 17 & 34.00 & \multirow[t]{2}{*}{0.53} & \multirow[t]{2}{*}{0.47} \\
\hline Female & 64 & 71.91 & 25 & 28.09 & & \\
\hline Level of Education & \multicolumn{2}{|l|}{$\mathrm{N}=95$} & \multicolumn{2}{|l|}{$\mathrm{N}=43$} & & \\
\hline None & 22 & 78.57 & 6 & 21.43 & \multirow[t]{4}{*}{4.06} & \multirow[t]{4}{*}{0.26} \\
\hline Primary & 21 & 75.00 & 7 & 25.00 & & \\
\hline Secondary & 44 & 64.71 & 24 & 35.29 & & \\
\hline Tertiary & 5 & 55.56 & 4 & 44.44 & & \\
\hline Vocational/Tech & 3 & 60.00 & 2 & 40.00 & & \\
\hline Professional Category & \multicolumn{2}{|l|}{$\mathrm{N}=91$} & \multicolumn{2}{|l|}{$\mathrm{N}=40$} & & \\
\hline Medical & 9 & 64.29 & 5 & 35.71 & \multirow[t]{4}{*}{4.06} & \multirow[t]{4}{*}{0.26} \\
\hline Nursing & 51 & 66.23 & 26 & 33.77 & & \\
\hline Laboratory Technician & 11 & 64.71 & 6 & 35.29 & & \\
\hline Paramedic & 11 & 9.17 & 3 & 13.04 & & \\
\hline Years of Experience & \multicolumn{2}{|l|}{$\mathrm{N}=92$} & \multicolumn{2}{|l|}{$\mathrm{N}=41$} & & \\
\hline $1-5$ years & 14 & 71.93 & 16 & 28.07 & \multirow[t]{4}{*}{1.20} & \multirow[t]{4}{*}{0.75} \\
\hline $6-10$ years & 33 & 64.71 & 18 & 35.29 & & \\
\hline $11-20$ years & 15 & 75.00 & 5 & 25.00 & & \\
\hline $20-40$ years & 3 & 60.00 & 2 & 40.00 & & \\
\hline Time constraint & \multicolumn{2}{|l|}{$\mathrm{N}=93$} & \multicolumn{2}{|l|}{$\mathrm{N}=41$} & & \\
\hline Always & 27 & 96.43 & 1 & 3.57 & \multirow[t]{3}{*}{30.43} & \multirow[t]{3}{*}{ 0.001* } \\
\hline Sometimes & 48 & 78.69 & 13 & 21.31 & & \\
\hline Never & 18 & 40.00 & 27 & 60.00 & & \\
\hline Knowledge & \multicolumn{2}{|l|}{$\mathrm{N}=97$} & \multicolumn{2}{|l|}{$\mathrm{N}=43$} & & \\
\hline Poor & 4 & 100.00 & 0 & 0.00 & \multirow[t]{3}{*}{2.19} & \multirow[t]{3}{*}{0.33} \\
\hline Adequate & 33 & 71.74 & 13 & 28.26 & & \\
\hline Excellent & 60 & 66.67 & 30 & 33.33 & & \\
\hline
\end{tabular}

*Significance level of $\mathrm{p}<0.05$. 


\section{Multivariate Logistic Regression Analysis: Association between Independent and Dependent Variables}

A multivariate logistic regression model was run between independent variables and the outcome variable (compliance with IPC). The multivariate analysis for the crude odds ratio found that health workers who never experienced time constraints at work were 40.5 times more likely to comply with IPC policy than those who always had time constraints. The adjusted odds ratio revealed that health workers who never had time constraints at work were 92.36 times more likely to comply with IPC guidelines than those who always had time constraints at work. Only the adjusted odds ratio found significance between the Level of Education and Compliance with IPC ( $p=$ 0.044). Respondents who had tertiary education were found to be 12.64 times more likely to comply with IPC policy than those who had no formal education. Results of the Crude and Adjusted Odds Ratios are shown in table 5 below.

Table 5. Multivariate Logistic Regression Analysis: Association between Independent Variables and Compliance with IPC Policy

\begin{tabular}{|c|c|c|c|c|}
\hline Variable & Crude OR (95\% CI) & P-value & Adjusted OR (95\% CI) & P-value \\
\hline \multicolumn{5}{|l|}{ Sex } \\
\hline Male (reference) & 1 & - & 1 & - \\
\hline Female & $0.76(0.36-1.60)$ & 0.467 & $0.63(0.19-2.15)$ & 0.466 \\
\hline Age & $1.01(0.97-1.06)$ & 0.643 & $1.08(0.93-1.25)$ & 0.317 \\
\hline \multicolumn{5}{|l|}{ Level of Education } \\
\hline None (reference) & 1 & - & 1 & - \\
\hline Primary & $1.22(0.35-4.24)$ & 0.752 & $0.93(0.13-6.58)$ & 0.945 \\
\hline Secondary & $2.00(0.71-5.61)$ & 0.187 & $5.29(0.30-10.19)$ & 0.198 \\
\hline Tertiary & $2.93(0.59-14.45)$ & 0.186 & $12.64(1.08-148.6)$ & 0.044* \\
\hline Vocational/Technical & $2.44(0.33-18.13)$ & 0.380 & $5.34(1.10-152.12)$ & 0.226 \\
\hline \multicolumn{5}{|l|}{ Professional Category } \\
\hline Medical (reference) & 1 & - & 1 & - \\
\hline Nursing & $0.92(0.28-3.02)$ & 0.888 & $1.50(0.22-10.31)$ & 0.683 \\
\hline Lab. Technician & $0.98(0.22-4.30)$ & 0.981 & $2.64(0.26-26.56)$ & 0.409 \\
\hline Paramedic & $0.27(0.12-0.05)$ & 1.38 & $0.14(0.14-1.34)$ & 0.088 \\
\hline \multicolumn{5}{|l|}{ Years of Experience } \\
\hline 1-5 years (reference) & 1 & - & 1 & - \\
\hline $6-10$ years & $1.40(0.62-3.16)$ & 4.20 & $0.89(0.28-4.02)$ & 0.881 \\
\hline $11-20$ years & $0.85(0.27-2.74)$ & 0.791 & $0.13(0.01-2.64)$ & 0.184 \\
\hline 20- 40 years & $1.71(0.26-11.20)$ & 0.580 & $0.04(0.001-7.81)$ & 0.229 \\
\hline \multicolumn{5}{|l|}{ Time constraints } \\
\hline Always & 1 & - & 1 & - \\
\hline Sometimes & 7.31(0.91-58.99) & 0.062 & $4.50(0.481-41.81)$ & 0.186 \\
\hline Never & $40.5(5.04-325.20)$ & 0.001* & $92.36(8.62-989.8)$ & 0.001* \\
\hline \multicolumn{5}{|l|}{ Knowledge } \\
\hline Poor (reference) & 1 & - & 1 & - \\
\hline Adequate & $0.79(0.36-1.71)$ & 0.548 & $1.07(0.29-3.93)$ & 0.919 \\
\hline Excellent & 1 (omitted) & - & 1 (omitted) & - \\
\hline
\end{tabular}




\section{Discussion}

\section{Health Worker Knowledge}

This study found that the overall level of knowledge of IPC guidelines among respondents was high, as $97 \%$ had adequate knowledge. This level of knowledge was high compared with a study in Zambia, in which the level of knowledge was $60.7 \%$ [13]. This high level of knowledge among health workers in Ghana could be due to the increased training and awareness creation about IPC protocols. For instance, the proportion of respondents who had had IPC training was $94.1 \%$. This rate was also high compared with $26.5 \%$ of respondents who had had IPC training in KL5 Hospital in Kenya [14] as well as $65.6 \%$ of participants who had had IPC training in MAG Hospital in Ethiopia [15].

The level of knowledge was also found to be low in other studies. For instance, only $57.8 \%$ of respondents knew that tetanus could be transmitted through dirty needles and sharps in Ethiopia (15). A study also found that only $32 \%$ of health workers had had any training on IPC, only $28 \%$ had excellent knowledge of IPC, and $50 \%$ had a moderate level of knowledge of IPC precautions [16]. Therefore, the indication is that regular IPC training has a direct positive impact on the level of knowledge of IPC protocols.

However, the study also revealed that $100 \%$ of respondents who had poor knowledge of IPC were non-compliant with IPC guidelines, $71.7 \%$ of those who had adequate knowledge, and $66.6 \%$ of those who had excellent knowledge were also non-compliant with IPC. A $100 \%$ non-compliance among respondents with poor knowledge confirms the finding that lack of awareness was a major factor for noncompliance [17].

Despite the important role of education or training in ensuring IPC compliance, a study also stated that education alone did not make health workers comply with IPC measures [18]. This assertion was confirmed by the fact that
$66.6 \%$ of respondents who had excellent knowledge of IPC were non-compliant with IPC policy guidelines. For this reason, [19] recommended that a dedicated team of IPC personnel is needed to provide surveillance, data collection, and oversee the implementation of IPC policy locally because education alone does not improve IPC practices.

\section{Availability of IPC Resources}

Availability of IPC resources were found to be low as less than one-third (31.4\%) of respondents always had basic IPC resources like soap, water, and hand towel available for use at work. On the other hand, the remaining majority (68.6\%) did not always have soap, water, and hand towels available. This finding agrees with the $76.6 \%$ of inadequate resource supply found in earlier studies [13]. Therefore, the indication was that the low availability of IPC resources was a widespread problem, especially in developing countries, and could be attributed to a lack of funds to procure the needed resources as documented in the literature [20].

This notwithstanding, the problem needs to be addressed because inadequate IPC resources have been found to be a barrier to IPC practice [13]. This is due to the fact that health workers need to use IPC resources in order to practice IPC standard precautions. However, it is worth noting that the results of this study showed that, although both the availability of IPC resources and IPC compliance were low, there was no significant association found between them.

\section{Health Workers' Compliance with IPC Policy Guidelines}

The study indicated that compliance with IPC guidelines among the health workers at the La General Hospital was low (30.7\%) despite the high level of knowledge of IPC guidelines (97\%). This finding was low compared with the finding of a study, in which compliance with hand hygiene was $61 \%$ and it was said to be moderate [21]. Contrary to this study's finding 
on compliance, a similar study at the Ridge Hospital currently, the Greater Accra Regional Hospital in Ghana revealed that both health workers' knowledge and compliance were $70.6 \%$ and $45 \%$, which were described as low and moderate, respectively [10].

In assessing the relationship between knowledge of IPC, availability of IPC resources, time constraint at work, and compliance with IPC guidelines, time constraint at work were found to have a significant impact on compliance. Among respondents who always had time constraints at work, 96.4\% were non-compliant with IPC guidelines, while $40 \%$ of those who never had time constraints at work were non-compliant.

Although more than $93 \%$ of respondents stated that they knew that patients could get infections from healthcare settings and that hand washing could prevent infection transmission, only $57 \%$ stated that they washed their hands before touching a patient. This confirms the assertion that most HCAIs are transmitted by health personnel as a result of failure to practice standard precautions such as hand hygiene and change of gloves between patient contacts [22]. In addition, the high level of health worker knowledge of IPC compared with the low IPC compliance in this study confirms the assertion that there was not enough proof that education has a long-term effect on IPC practice [18]. Another finding, which is worth noting is that whereas $57 \%$ of respondents wash their hands before touching patients, $84.9 \%$ wash their hands after touching patients giving the impression that health workers were not as precautious about what they could transfer to patients as they were about what they could contract from patients. It also confirms the finding that health workers practice hand hygiene less than half the time they should, even though they know the importance of hand hygiene [23].

\section{Time Constraint at Work}

Time constraints at work were found to have influenced the ability of health workers to adhere to IPC guidelines. Among the participants, $66.4 \%$ admitted that time constraint at work was a barrier to their practice of hand hygiene. Although this finding was high, it agreed with a study where $26 \%$ of participants indicated that lack of time was one of the factors that impeded proper infection prevention and control practices [16]. A similar finding had been noted that compliance of health workers with IPC guidelines improved from $25 \%$ when there was a shortage of staff to $70 \%$ when staffing improved to normal [24].

This was attributable to the fact that some health workers perceived IPC standard precautions as an extra workload instead of it being a vital part of patient safety and quality care [25]. According to some health personnel, it was time-consuming to adhere to all IPC guidelines, such as washing hands before and after each contact with each patient, changing gloves between patient contacts, separating the waste into various categories, and so on. In their report, [24] added that these IPC guidelines tend to compete with other health care delivery practices for the health worker's time and often end up not observed when workload increases or there is a shortage of staff.

The chi-square test results of this study showed that time constraint at work was significantly associated with compliance with IPC precautions among health workers. The multivariate analysis for the crude odds ratio found that health workers who never experienced time constraints at work were 40.5 times more likely to comply with IPC policy than those who always had time constraints. The adjusted odds ratio revealed that health workers who never had time constraints at work were 92.36 times more likely to comply with 
IPC guidelines than those who always had time constraints at work. This confirmed the assertion that limited health worker time at work impedes their ability to adhere to IPC guidelines [13]. This may account for the difference in compliance between departments since the tasks of health delivery vary among departments. In addition, the results revealed that health workers who had a tertiary level of education were 12.64 times more likely to comply with IPC policy than those with no level of formal education.

\section{Equations}

The equation used to determine the sample size was based on Cochran's (1977) formula, as shown below.

$$
\left.\mathrm{n}=\mathrm{z}^{2}(\mathrm{pq})\right) \mathrm{d}^{2}
$$

\section{[1] Cochran's formula [26].}

Using this formula, the total number of health workers at the La General Hospital was 421, and the obtained sample size was 134 .

A nonresponse rate of $7 \%$ was calculated and added to the obtained sample size to cater for questionnaires that may not be answered and returned. Therefore 9 questionnaires were added to the 134, and 143 questionnaires were given out.

\section{Limitation}

Because participants could respond to questions with answers, which were different from what they actually practiced, the addition of an observation component to the selfadministered questionnaire for data collection would have enabled a comparison to be made between the written down answers and the actual ongoing practices. However, this could not be done due to the limitation of time. This notwithstanding, the results of this study are applicable to the general/target population.

\section{Further Research Works}

Future research that could be carried out based on the outcome of this study includes interdepartmental IPC compliance within the same health care facility in order to determine the extent to which factors like workload affect health worker compliance. This would bring out any variations in the rate of compliance associated with the variations in the nature of work, workload, and staffing in different departments of the same health facility and under the same management.

\section{Conclusion}

The study was set out to examine factors associated with infection prevention and control (IPC) policy/guidelines in Ghana. This was achieved by conducting a survey among health workers at a municipal hospital in the Greater Accra Region. The study concludes that health workers' knowledge of IPC was high, which was in agreement with the percentage of health workers who had attended IPC trainings and workshops organized by the IPC Committee of the hospital. The study found that efforts were being made to disseminate the IPC policy. However, non-compliance as a result of time constraints at work was also high. Availability of IPC resources as well as health workers' compliance with IPC guidelines was found to be low. Based on the findings of this study, it is recommended that policy makers and practitioners in the health sector of Ghana and other countries with similar resource constraints should seek to put in place measures that would enhance health workers' compliance with IPC. This would help assuage the problem of crossinfection, and hospital-acquired infections among health workers.

\section{Acknowledgement}

My first acknowledgement goes to God Almighty, who has made it possible for me to carry out this work. I acknowledge my husband, Mr. Albert Wornyo, for his support, which he provided to me throughout this program. Again, I acknowledge my supervisor, Prof. Justice Non-vignon, as well as all my lecturers, who provided me with the needed guidance in the process of carrying out this 
work. Acknowledgement also goes to Madam Evelyn, the IPC coordinator of La General Hospital, and Mr. Charles Amankwa for their assistance during the data collection process at the hospital. Finally, I acknowledge my family and Pastor Christian Avornyo for constantly encouraging me to persevere amidst all the

\section{Reference}

[1] Gracia-Zapata, M. R., Silva e Souza, A. C., Guimaraes, J. V., Tipple, A. F. V., Prado, M. A., Gracia-Zapata, M. T. A., 2010, Standard precautions: knowledge and practice among nursing and medical students in a teaching hospital in Brazil. International Journal of Infection Control, 6(1). DOI: https://doi.org/10.3396/ijic.v6i1.4075.

[2] Jackson, C., Lowton, K., and Griffiths, P., 2014, Infection Prevention as a 'Show': A Qualitative Study of Nurses' Infection Prevention Behaviors. International Journal of Nursing Studies, 51(3), 400-408.

[3] WHO, 2010, The burden of health care associated infections World-wide Geneva, Switzerland: $\quad$ WHO http://www.who.int/gpsc/country_work/burden_hcai /en/.

[4] Public Health England, 2014, Health care associated infections (HCAI): guidance, data and analysis. Accessed on 14/5/2018. www.gov.uk/government/collections/.

[5] National collaborating centre for women's and children's health (UK), 2008, Surgical Site Infection: Prevention and Treatment of Surgical Site Infection. (London, U.K.: National Institute of Clinical Excellence (NICE)). https://www.ncbi.nih.gov/books/NBK53731/. [6] Abou El-Enein, N. Y., \& El-Mahdy, H. M., 2011, Standard precautions: a knowledge, attitude and practice study among nurses in the dialysis unit in a University Hospital in Alexandria, Egypt. Journal of Egypt Public Health Association, 86, 310.

[7] WHO, 2016, Health care associated infections fact sheet Accessed on 14/5/2018 http://tinyurl.com/d2qwn9m. challenges I encountered at the time of carrying out this work.

\section{Conflict of Interest}

The authors declare that there is no conflict of interest regarding this study.

[8] Malliarou, M., Sarafis, P., Zyga, S. \& Constantinidis, T., 2013, The importance of nurse hand hygiene. International Journal of Caring Sciences, 6(3), 327-331.

[9] Ministry of Health, Ghana, (National Policy and Guidelines for Infection Prevention and Control in Health Care Settings in Ghana, 2015), Accessed on $15 / 12 / 2017$.

http://www.ghanahealthservice.org/downloads/Natio nal_Policy_and_Guidelines\%20_for_Infection_Prev ention_and_Control_in_Health_Care_Settings_2015 .pdf.

[10] Hayeh, P. A., 2012, Infection Prevention and Control Practices Among Health Workers at Ridge Regional Hospital. (Master's Thesis) University of Ghana.

http://197.255.68.203/handle/123456789/5874 and http://ugspace.ug.edu.gh/handle.

[11]Enemark, U., Newman, M., Asante, F., \& Fenny, A., 2016, Healthcare Associated Infections in Ghana. Accessed on 20/2/2018. Available at http://drp.dfcentre.com/project/healthcare-

associated-infections-ghana and http://haiproject.org.

[12] Myjoyonline, 2017, Third time lucky: man shares harrowing experience after losing 2 babies at KATH Accessed on 13/6/2018. Available at https://www.myjoyonline.com/.

[13] Njovu, E., 2016, Factors affecting compliance to Infection Prevention and Control guidelines by Nurses at St. Dominic Mission Hospital, Ndola Copperbelt, Zambia. Texila International Journal, 2(2), 1-10.

[14] Maosa, E. K., 2012, Factors influencing hospital infection prevention and control practices among medical staff in Kisii Level Five Hospital, Kisii 
County, Kenya. (Master's Thesis), The University of Nairobi, Kenya.

http://erepository.uonbi.ac.ke/handle.

[15] Yakob, E., Lamaro, T., \& Henok, A., 2015, knowledge, attitude and practice towards infection Control measures among Mizan- Aman General Hospital Workers, South West Ethiopia. Journal of Community Medicine and Health Education, 5(5), 370. Doi: 10.4172/2161 -0711.1000370.

[16] Chipfuwa, T., Manwere, A., \& Shayamano, P., 2014, Barriers to infection prevention and control (IPC) practice among nurses at Bindura Provincial Hospital, Zimbabwe. IOSR Journal of nursing and health science, 3(1), 69-73.

[17] Suchitra, J. B., \& Lakshmi Devi, N., 2007, Impact of education on knowledge, attitudes and practices among various categories of health care workers on nosocomial infections. India Journal of Medical Microbiology.25(3), 181-187.

[18] Ward, D., 2011, The role of education in the prevention and control of infection: A review of the literature Nurse Education Today. 31(1), 9-17.

[19] Warren, D., \& Kollef, M., 2005, Prevention of Hospital Infection. Microbes and Infection. 7(2), 267-273.

[20]Raka, L., 2010, prevention and control of hospital-related infections in low- and middleincome Countries. The Open Infectious Disease Journal. 4(1), 125 - 131.
[21] Mukwato, K. P., Ngoma, C. M., \& Maimbolwa, M., 2007, compliance with infection prevention guidelines by health care workers at Roland Ross General Hospital Mufulira District. Medical Journal of Zambia, 35(3), 110-116.

[22] Delaune, S. C., \& Ladner, P. K., 2010, Fundamentals of nursing standards and practice (4th ed.) (Clifton Park, NY: USA Cengage Learning).

[23] Canadian Committee on Antibiotic Resistance, 2007, Infection Prevention and Control Best Practices for Long Term Care, Home and Community Care including Health Care Offices and Ambulatory Clinics. Accessed on 10/3/2018. www.ccar-ccra.org.

[24] Harbarth, S., Sudre, P., Dharan, S., Cadenas, M. \& Pittet, D., 1999, Outbreak of Enterobacter Clocae Related to Understaffing, overcrowding and Poor Hygiene Practices. Infection Control and Hospital Epidemilogy, 20(9), 598-603.

[25] Ward, D. J., 2012, Attitudes towards infection prevention and control: an interview study with nursing students and nurse mentors. PubMed Journal, 21(4):301-6. Doi: 10.1136/bmjqs-2011000360 PMID:22328459.

[26] Israel, G. D. (1992). Determining sample size. University of Florida Cooperative Extension Service http://www.sciepub.com. 